\title{
Unilateral diaphragmatic paralysis: an electrophysiological study
}

\author{
A Lagueny, E Ellie, J Saintarailles, R Marthan, M Barat, J Julien
}

\begin{abstract}
An electrophysiological study was carried out on four patients with unilateral diaphragmatic paralysis. Whereas neurogenic involvement of the paralysed hemidiaphragm was roughly similar in all cases, neurogenic patterns could be detected in the normally moving controlateral hemidiaphragm in three cases, and the degree of involvement could be correlated with the respiratory state of the patients. EMG also showed that the neuropathic process affected the limb muscles. Thus unilateral diaphragmatic paralysis may be, at least in some cases, the localised expression of a more diffuse neuropathy, perhaps a peculiar form of neuralgic amyotrophy.
\end{abstract}

Diaphragmatic paralysis has been attributed to multiple causes, ${ }^{12}$ the most common being a tumour affecting the phrenic nerve. In some cases, however, the cause remains unknown and the paralysis is then called idiopathic. ${ }^{13-5}$ Bilateral diaphragmatic paralysis induces dyspnoea on exertion and supine position, ${ }^{2}$ whereas unilateral diaphragmatic paralysis may be asymptomatic and discovered only in routine chest $x$ ray pictures. We carried out an electrophysiological study on four patients suffering from apparently unilateral idiopathic diaphragmatic paralysis. The paralysis was both isolated and complete on the chest $x$ ray picture in all of the patients, although the respiratory complaints differed from case to case. Our aim was to determine whether the results of the electrophysiological tests could explain this disparity and investigate the mechanism of the paralysis.

\section{Patients and methods}

ELECTROPHYSIOLOGICAL METHODS

The tests were performed with MS6 (Medelec) or Viking (Nicolet) EMG apparatus. The patients were in a supine position. To record EMG a $7 \mathrm{~cm}$ long coaxial needle was inserted below the xiphoid appendix through the rectus abdominus and pushed along the posterior border of the sternum up to the sternal insertions of the right or left hemidiaphragm (RHD and LHD). The needle was left in place and conduction along the phrenic nerve was measured by stimulating the nerve with a surface electrode located at the lateral edge of the sternocleidomastoid. The latencies of the compound muscle action potential (CMAP) from the RHD and LHD after right and left phrenic nerve stimulation respectively, were measured and compared (normal range 6-8 ms). When the EMG showed motor unit potentials (MUPs) during deep inspiration in the paralysed HD but no CMAP after ipsilateral phrenic nerve stimulation, the controlateral phrenic nerve was stimulated to look for a possible crossed innervation. When we failed to record activity from the diaphragm with this method the coaxial needle was inserted under radioscopic control on the middle axillary line, between the seventh and eighth intercostal spaces and pushed up to the costal insertion of the diaphragm. ${ }^{6}$ The CMAP after phrenic nerve stimulation was recorded as before. During these investigations no complications were observed, and coaxial needles gave information on MUPs and interference pattern that could not be obtained from surface or oesophageal electrodes. As a rule we recorded the EMG of limb muscles and looked for activity at rest in the cervical paravertebral muscles. Motor and sensory nerve conduction velocities (MNCVs and SNCVs, respectively) and short somatosensory evoked potentials (SSEPs) were also studied.

\section{CASE REPORTS}

Case 1 In January 1987 a 32 year old man had an isolated paralysis of the LHD discovered on routine chest $x$ ray pictures. By March 1988 he was asymptomatic, and neurological examination and lung function tests yielded normal results. EMG showed a single unit pattern in the paralysed RHD and a normal pattern in the LHD (fig 1). Intermediate patterns with large MUPs were recorded in the right biceps brachii and deltoid. The CMAP evoked in the RHD was delayed $(10.6 \mathrm{~ms})$ whereas the CMAP latency was normal in the LHD (fig 2). We re-examined the patient one and two years later. Although the RHD was still denervated, the EMG patterns in the right deltoid and biceps had returned to normal.

Case 2 In July 1987 a 68 year old man experienced sudden breathlessness on exertion and complained of moderate pain in the right shoulder and in the neck. Chest $x$ ray pictures showed an elevated and motionless RHD. One year before he had suffered from pain and 
Figure 1 EMG traces of needle examination of diaphragm in patient 1. During each inspiration a single unit pattern is recorded in paralysed hemidiaphragm [a-b] and a normal pattern is recorded in contralateral hemidiaphragm [c-d] (calibration $a$ and $c$ : (calibration a and $c$ :
vertical bar $500 \mu \mathrm{V}$, horizontal bar $500 \mathrm{~ms}$; $b$ and d: vertical bar $1 \mathrm{mV}$, horizontal bar $50 \mathrm{~ms}$ ).

Figure 2 Compound muscle action potential [CMAP] recorded in diaphragm by stimulating phrenic nerve at neck in patient 1. Two traces for each site of recording; $A 1$ and $A 2$ show delayed latency and low polyphasic CMAP in paralysed $H D$ whereas B4 and B5 show normal latency and normal CMAP in contralateral HD (calibration: horizontal bar $5 \mathrm{~ms}$, vertical bar $500 \mu \mathrm{V}$ ).
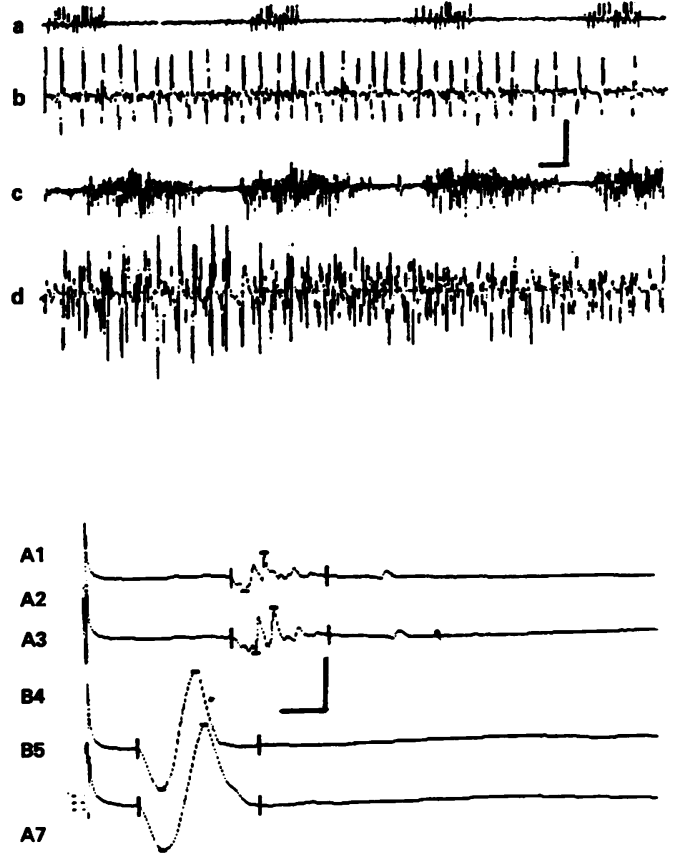

weakness in both shoulders for one month, which had disappeared spontaneously. In January 1988 the RHD was still paralysed, and the patient experienced moderate dyspnoea in the supine but not standing position. Neurological examination was normal. Lung function tests showed a moderate distal obstruction that reduced the forced end expiratory flow rates without restriction. Maximal alveolar pressure during forced expiration ( $\mathrm{P}$ Alv Max) was $79 \mathrm{kPa} .{ }^{7}$ EMG showed a single unit pattern in the paralysed RHD and an incomplete interference pattern in the LHD, the right deltoid, and biceps. No CMAP could be evoked in the RHD. On the left the CMAP latency was slightly prolonged $(9.3 \mathrm{~ms})$. At follow up examinations, 12 and 22 months later, clinical and electrophysiological data were unchanged.

Case 3 In August 1988 a 49 year old man suddenly complained of dyspnoea on climbing up stairs. The chest $x$ ray picture showed an isolated paralysis of the RHD. In November 1988 his neurological examination was normal. Lung function tests showed a restrictive syndrome. P Alv Max was $71 \mathrm{kPa}$. An EMG under fluoroscopic control showed no potential in the RHD and an incomplete interference pattern in the LHD. There was an incomplete interference pattern with large MUPs in the intercostal muscles, deltoid, biceps, first dorsal interosseous, tibialis anterior on the right, and deltoid on the left. No CMAP could be recorded in the RHD. In the LHD the CMAP latency was prolonged $(9.4 \mathrm{~ms})$. Two years later only a moderate dyspnoea on exertion persisted. An EMG showed a single unit pattern in the RHD, but no CMAP could be evoked. In the LHD the interference pattern remained incomplete and the CMAP latency was normal.
Case 4 In September 1983 a 56 year old man was admitted for sudden dyspnoea and pain located at the cervico-dorsal junction. Chest $x$ ray pictures showed an isolated paralysis of the LHD. In February 1985 he complained of dyspnoea in the supine position and breathlessness on walking. Scarce fasciculations in the deltoids and biceps, a slight motor deficit of the scapular girdle and brisk tendon reflexes were noted. Lung function tests showed a restrictive syndrome with hypoxaemia. P Alv Max was $86 \mathrm{kPa}$. An EMG showed a single unit pattern in the paralysed LHD and a severe incomplete pattern in the RHD. No CMAP could be evoked in the LHD, while on the right the CMAP latency was delayed $(11 \mathrm{~ms})$. Intermediate patterns with large MUPs were found in the deltoid, biceps, and infra spinatus on both sides. Amyotrophic lateral sclerosis (ALS) was suspected. One year later his clinical condition and the results of the electrophysiological investigation of the diaphragm were unchanged. More MUPs, however, were recruited at full effort in the deltoids and biceps, although the EMG patterns were still considered as neurogenic. The patient's condition remained stable for about three years and afterwards he slightly recovered. In March 1990 his neurological examination was normal except for brisk reflexes. Follow up allowed us to exclude ALS.

For all of the patients biological investigations yielded negative results. MRI of the spinal cord (cases 3 and 4), bronchoscopy, CSF examination, and cervico-mediastinal CT scan (cases 1 and 2) were normal. In case 4 the CSF protein content was $0.8 \mathrm{~g}$, with normal cell count and gammaglobulin concentration. Muscle biopsy specimens from the right (case 3) and left deltoid (case 4) showed a moderate denervation atrophy. We did not record activity at rest in the cervical paravertebral muscles. MNCVs, SNCVs, sensory nerve action potential amplitudes, and N20 latency of SSEPs by stimulating the right median nerve at the wrist were normal. F-wave latencies were within a normal range, except for patient 4 (left median nerve, F-wave latency $=31 \mathrm{~ms}$, normal $<30$ $\mathrm{ms}$; and left tibial nerve, $\mathrm{F}$-wave latency = $63 \mathrm{~ms}$, normal $<60 \mathrm{~ms}$ ).

\section{Discussion}

Unilateral idiopathic diaphragmatic paralysis is more common in men and mainly affects the RHD. ${ }^{1}$ Onset is usually abrupt with sudden dyspnoea, although the paralysis is sometimes discovered on routine chest $x$ ray pictures. Two of our cases had pain located in the neck or in the shoulder ipsilateral to the paralysis. Pain, especially of the shoulder region, may reveal phrenic nerve paralysis. ${ }^{8}$ The prognosis is good, and even though for many patients the paralysis is permanent, few complain of exertional dyspnoea. ${ }^{14}$

Although all of our patients had unilateral diaphragmatic paralysis in chest $x$ ray pictures, the results of the pulmonary function tests and the severity of the dyspnoea clearly differed from one to another. The severity of the 
electrophysiological involvement of the paralysed HD was roughly similar; EMG showed single unit patterns or the absence of MUPs, and no CMAP could be obtained by stimulating the phrenic nerve (except on the first examination of patient 1). By contrast, in the contralateral HD the results of the electrophysiological tests were different and may correlate with respiratory state. Even though the contralateral HD moved normally the patients who were dyspnoeic had neurogenic patterns of incomplete interference and slightly delayed CMAP latencies. Cases 3 and 4 who both had a restrictive ventilatory defect exhibited a severe neurogenic involvement and delayed CMAP latencies, whereas case 1, whose pulmonary function tests were normal, had normal interference pattern and CMAP latency in the contralateral moving $\mathrm{HD}$.

EMG also demonstrated that the neurogenic alterations were not limited to the diaphragm. In cases 1 and 2 incomplete interference patterns were recorded in the proximal muscles of the upper limb ipsilateral to the diaphragmatic paralysis, whereas in cases 3 and 4 abnormal patterns were even more widespread, involving both shoulder girdles and in one case, the lower limb. These results suggest that in unilateral idiopathic diaphragmatic paralysis, systematic EMG of the contralateral HD and of some proximal and distal limb muscles should be performed to detect a possible subclinical denervation.

These cases of unilateral diaphragmatic paralysis share common aspects with neuralgic amyotrophy, ${ }^{9}$ characterised in its typical form by the sudden onset of shoulder pain followed by motor weakness and atrophy and an overall good prognosis. Our patients, who presented with apparently unilateral idiopathic diaphragmatic paralysis, had an acute, partly regressive, and electrophysiologically widespread neuropathy which involved both hemidiaphragms (except case 1). Painless forms of neuralgic amyotrophy have been reported, ${ }^{10}$ and although none of our patients presented with the typical acute pain of the shoulder, EMG clearly showed that at least some proximal muscles of the arm ipsilateral to the diaphragmatic paralysis were affected. Even though diaphragmatic paralysis is rare in neuralgic amyotrophy, 27 cases were listed by Walsh et al. ${ }^{11}$ Gregory et al reported a patient who experienced recurrent isolated phrenic nerve paralyses, and suggested that recurrent diaphragmatic paralysis might represent a mononeuropathic variant of brachial neuritis. ${ }^{12}$ England and Sumner recently stressed that neuralgic amyotrophy may be a considerably more diverse entity than generally appreciated. ${ }^{13}$ Our results suggest that, at least in some cases, unilateral idiopathic diaphragmatic paralysis may be a peculiar form of neuralgic amyotrophy. The basic mechanism remains unknown but the length of the phrenic nerve may render it more liable to some factors (repetitive trauma, immunisation) putatively implicated in the pathogenesis of the disease.

1 Riley EA. Idiopathic diaphragmatic paralysis. A report of eight cases. Am $\mathcal{Y}$ Med 1962;32:404-16.

2 Chan CK, Loke J, Virgulto JA, Mohsenin V, Ferranti R, Lammertse T. Bilateral diaphragmatic paralysis: clinical spectrum, prognosis and diagnostic approach. Arch Phys Med Rehabil 1988;69:976-9.

3 Aleksic SN, Lieberman A, George AE. Idiopathic diaphragmatic paralysis and neuralgic amyotrophy: two aspects of the same disorder. Europ Neurol 1973;10:243-9.

4 Douglass BE, Clagett OT. The prognosis in idiopathic diaphragmatic paralysis. Dis Chest 1960;37:294-7.

5 Lieberman A. Spontaneous unilateral diaphragmatic paresis. NY State $\mathcal{F}$ Med 1954;54:2737.

6 Isch-Treussard C, Pauli G, Jesel M, Miech G, Isch F. Etude électromyographique des paralysies hémidiaphragmatiques. Rev EEG Neurophysiol 1974;4:467-75.

7 Kays C, Guenard H, Choukroun ML. Pression alvéolaire pendant la capacité vitale. Bull Europ Physiopath Resp 1982;18:501-13.

8 Ross GS, Wolf JK, Chipman M. Neuralgias. In: Baker AB Joynt RJ, eds. Clinical neurology. Philadelphia: Harper and

9 Parsonage MJ, Turner JWA. Neuralgic amyotrophy; the arsonage MJ, Turner JWA. Neuralgic amyotrophy;
shoulder girdle syndrome. Lancet 1948;254:973-8.

10 Schott GD. A chronic and painless form of idiopathic brachial plexus neuropathy. $\mathcal{f}$ Neurol Neurosurg Psychiatry 1983;46:555-7.

11 Walsh NE, Dumitru D, Kalantri A, Roman AM. Brachial neuritis involving the bilateral phrenic nerves. Arch Phys Med Rehabil 1987;68:46-8.

12 Gregory RP, Loh L, Newsom-Davis J. Recurrent isolated alternating phrenic nerve palsies: a variant of brachial neuritis. Thorax 1990;45:420-1.

13 England JD, Sumner AJ. Neuralgic amyotrophy: an increasingly diverse entity. Muscle Nerve 1987;10:60-8. 